\title{
Magnetic traveling-stripe forcing: Enhanced transport in the advent of the Rosensweig instability
}

\author{
Thomas Friedrich, Ingo Rehberg, and Reinhard Richter \\ Experimentalphysik V, Universität Bayreuth, D-95440 Bayreuth, Germany
}

(Received 26 April 2010; published 3 September 2010)

\begin{abstract}
A contactless pumping mechanism is realized in a layer of ferrofluid via a spatiotemporally modulated magnetic field. The resulting pressure gradient leads to a liquid ramp, which is measured by means of x-rays. The transport mechanism works best if a resonance of the surface waves with the driving is achieved. The behavior can be understood by considering the magnetically influenced dispersion relation of the fluid.
\end{abstract}

DOI: 10.1103/PhysRevE.82.036304

PACS number(s): 47.20.Ma, 05.60.Cd, 47.35.Tv, 47.65.Cb

\section{INTRODUCTION}

Ferrofluids are colloidal dispersions of magnetic nanoparticles, which show superparamagnetic behavior [1]. This offers the advantage to control and facilitate their flow by timedependent magnetic fields. For example in alternating fields a viscosity reduction was predicted for Poiseuille flow [2] and thereafter experimentally confirmed [3,4]. More spectacularly, in rotating fields a spontaneous spin up of surface flow was observed in a beaker [5] and quantitatively analyzed in a circular duct [6].

An intriguing alternative to rotating fields is to apply a spatiotemporally modulated field. Here, one has to discern two transport mechanisms. One is "ferrohydrodynamic pumping" which was lately studied for a closed channel geometry $[7,8]$. It relies on the phase shift between the external field and the magnetization and works best for frequencies in the range of the inverse Brownian relaxation time, i.e., in the kilohertz regime. The second mechanism relies on traveling surface waves, driven by a traveling magnetic field. This was realized by an array of solenoids acting on a free surface of ferrofluid [9] and discussed in connection with the effect of peristaltic pumping [10]. As we will show in this paper the driving caused by a free surface gains momentum if the traveling field is superimposed by a constant field oriented normally to the fluid layer.

In such a vertically oriented magnetic field, a plain layer of ferrofluid transforms into a hexagonal array of crests if a critical threshold $H_{c}$ is overcome-the so-called Rosensweig instability [11]. Approaching $H_{c}$ from below, a vanishing of the phase velocity $v_{p}$ of surface waves was observed and explained by the nonmonotonic dispersion relation [12]. In this regime, a traveling-stripe forcing of the magnetic induction was predicted to excite a resonance of the wave amplitudes if the driving velocity coincides with $v_{p}$ [13]. This was recently verified by experiments [14]. As we show in the following, this resonance is correlated with a maximal net force_-as indicated by a liquid ramp_ on the magnetic fluid.

\section{EXPERIMENTAL SETUP AND PROCEDURE}

Our setup is sketched in Fig. 1. A Helmholtz pair of coils provides an applied field $H_{0}$ along the vertical direction by means of a stabilized electrical current. In the center of the coils a container machined from Perspex ${ }^{\mathrm{TM}}$ is located. It contains a box-shaped cavity with a length of $120 \mathrm{~mm}$, a width of $100 \mathrm{~mm}$, and a height of $25 \mathrm{~mm}$. This cavity is filled with $30 \mathrm{ml}$ of ferrofluid EMG909 (Table I). About $5 \mathrm{~mm}$ below the bottom of the cavity, a motor-driven conveyor belt is located. It harbors a grid of rods made from welding wire $(\varnothing$ $=2 \mathrm{~mm})$ with a spacing of $\lambda_{G}=(9.3 \pm 1) \mathrm{mm}$.

Radiation emitted by an x-ray tube mounted $1.5 \mathrm{~m}$ above the container is permeating the ferrofluidic layer and is recorded by an X-ray detector (16 bits). The absorption pictures serve to reconstruct the surface topography of the liquid layer. The measurement of the height is calibrated by means of a ferrofluidic ramp $[15,16]$. To eliminate the shadows cast by the rods we measure the time-averaged absorption of $\mathrm{x}$ rays with the moving belt included.

The belt leads to a spatial modulation of the magnetic field. The modulation of this field was measured within the empty cavity at one fixed position $2 \mathrm{~mm}$ above the ground of the container with the belt in motion. A result for a belt velocity $v_{G}$ of $0.75 \mathrm{~cm} / \mathrm{s}$ and an applied field of $\mu_{0} H_{0}$ $=22.29 \mathrm{mT}$, which has been measured by the Hall probe in the absence of the grid, is depicted in Fig. 2. The fit to a harmonic function demonstrates that the magnetic field can be modeled as a harmonic wave,

$$
H_{G}=H_{G, 0}+\Delta H_{G} \sin \left(\omega t-k_{G} x\right) .
$$

Here, $H_{G, 0}$ denotes the mean value and $\Delta H_{G, 0}$ denotes the amplitude of the modulated field. The frequency $\omega$ $=2 \pi v_{G} / \lambda_{G}$ [14] is controlled by the motor (Mattke MDR230). The wave number $k_{G}=2 \pi / \lambda_{G}$ is set by the grid spacing and has been chosen to be close to the critical wave number of the Rosensweig instability. The fact that $H_{G, 0}$ (gray dashed line) is slightly above the value of the applied field $H_{0}$ (gray

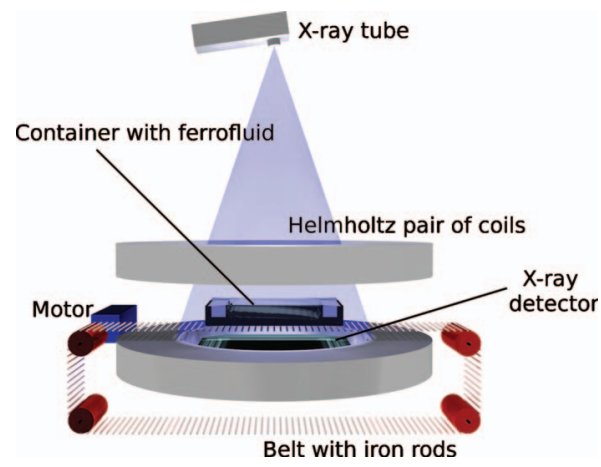

FIG. 1. (Color) Sketch of the experimental setup. 
TABLE I. Parameters measured for the ferrofluid EMG909 Lot H030308A from Ferrotec Co.

\begin{tabular}{lcc}
\hline \hline Density & $\rho$ & $0.9945 \mathrm{~g} / \mathrm{cm}^{3}$ \\
Surface tension & $\sigma$ & $23.37 \mathrm{mN} / \mathrm{m}$ \\
Initial susceptibility & $\chi_{0}$ & 0.95 \\
Viscosity & $\eta$ & $3.3 \mathrm{mPa} \mathrm{s}$ \\
Critical wavelength & $\lambda_{\mathrm{c}}=2 \pi \sqrt{\frac{\sigma}{\rho g_{0}}}$ & $9.89 \mathrm{~mm}$ \\
Calculated critical field & $\mu_{0} H_{\mathrm{c}, \text { calc }}$ & $21.35 \mathrm{mT}$ \\
Measured critical field & $\mu_{0} H_{\mathrm{c}, \text { meas }}$ & $21 \mathrm{mT}$ \\
\hline \hline
\end{tabular}

line) is explained by a focusing of the magnetic field toward the grid. About $30 \%$ of the area within the Helmholtz pair of coils is covered by the moving grid. This causes an increase in the average magnetic field strength $H_{G, 0}$ within the grid area since the field lines are attracted by the iron rods.

Figure 3 shows the modulation amplitude $\Delta H_{G}$ as a function of the applied magnetic field $H_{0}$. The fitted gray line indicates a linear dependence, thus demonstrating that the soft ferromagnetic material chosen for the rods is well suited for the experiments.

The modulation $\Delta H_{G}$ decreases with increasing distance from the rods. This is demonstrated experimentally in Fig. 4. The gray line is the fit to

$$
\Delta H_{G}(z)=\Delta H_{G}(0) e^{-z / \lambda_{G}},
$$

because we expect an exponential decay with the decay length given by the grid spacing $\lambda_{G}$ when assuming a twodimensional field. The fit yields $\mu_{0} \Delta H_{G}(0)=0.62 \mathrm{mT}$ for the modulation at the bottom of the cavity.

The ferrofluid EMG909 is made of magnetite particles dispersed in kerosene. Its nonlinear magnetization curve is plotted in Fig. 5. We measured $M(H)$ using a fluxmetric magnetometer consisting of a Helmholtz pair of sensing coils with 6800 windings and a commercial integrator (Lakeshore Fluxmeter 480). The sample is held in a spherical cavity with a diameter of $12.4 \mathrm{~mm}$ in order to provide a homogeneous magnetic field inside the sample with a demagnetization factor of $\frac{1}{3}$. The gray line is a fit using the Langevin function [1]

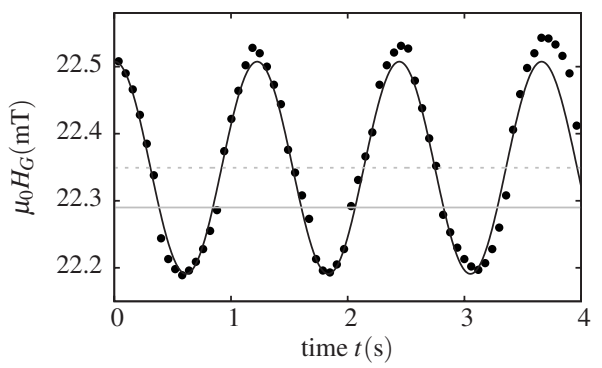

FIG. 2. The solid circles indicate the magnetic field as a function of time. The horizontal gray line corresponds to the applied field of the Helmholtz coils $\mu_{0} H_{0}$ as explained in the text. The black line represents a fitted harmonic function. The horizontal dashed gray line indicates the mean value $\mu_{0} H_{G, 0}$ of that fit.

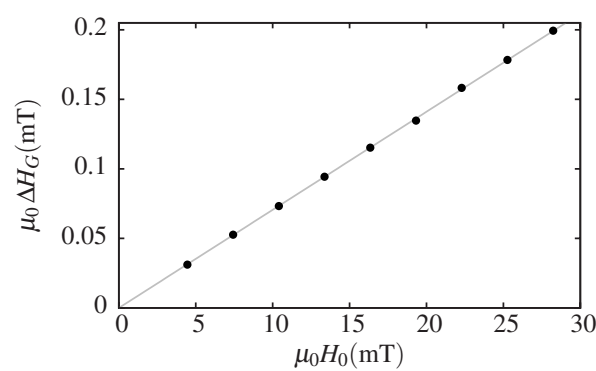

FIG. 3. Modulation amplitude vs the induction impressed by the coils. The experimental data (black solid circles) are recorded for a distance of $z=2 \mathrm{~mm}$ between the Hall probe and the ground of the container. Gray line: linear fit.

$$
M=M_{s}\left(\operatorname{coth}(\beta H)-\frac{1}{\beta H}\right) .
$$

This function was derived for dilute monodisperse colloidal suspensions and shows visible deviations from the data. In contrast, the black line displays a fit of a model for dense polydisperse magnetic fluids, namely, Eq. (32) of Ref. [17]. This model assumes a $\Gamma$ distribution for the particle diameter d,

$$
g(d)=\frac{1}{\Gamma(\alpha+1) d}\left(\frac{d}{d_{0}}\right)^{\alpha} \exp \left(-\frac{d}{d_{0}}\right),
$$

where $d_{0}=1.4 \mathrm{~nm}$ and $\alpha=3.8$ are the obtained fit parameters, corresponding to a mean particle diameter $\bar{d}=6.6 \mathrm{~nm}$. The fit also provides the volume fraction of magnetite to be $4.0 \%$.

Furthermore we characterize our ferrofluid by subjecting it to a homogeneous magnetic field, i.e., we omit for this measurement the driving belt. The plain layer becomes unstable at a critical field of $\mu_{0} H_{\mathrm{c}, \text { meas }}=21 \mathrm{mT}$. Like in Refs. $[16,18]$ this value was obtained by fitting the measured spike amplitudes with a scaling law provided by theory [19].

Figure 6(a) shows the surface topography of the magnetic liquid as reconstructed from the x-ray absorption, for a driving velocity of $v_{G}=4.1 \mathrm{~cm} / \mathrm{s}$ to the left-hand side. We averaged over 1300 subsequent images with an exposure time of $0.4 \mathrm{~s}$ each. Obviously the liquid builds up a ramp in the container. A longitudinal cut of the ramp is presented in Fig. 6(b) by black solid circles. Each data point stems from an average along the $y$ dimension selecting the innermost 36.4

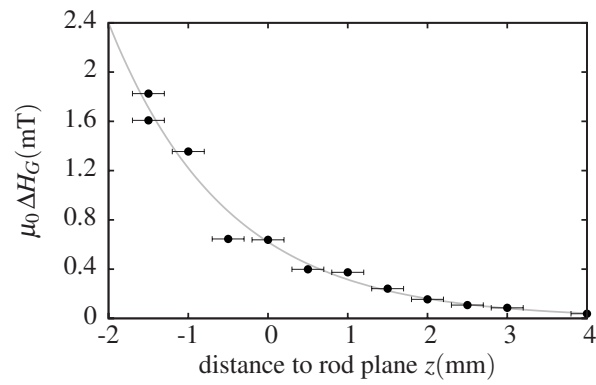

FIG. 4. $\Delta H_{G}$ measured for different distances $z$ at $\mu_{0} H_{0}$ $=22.29 \mathrm{mT}$ (black solid circles); gray line: exponential fit. 


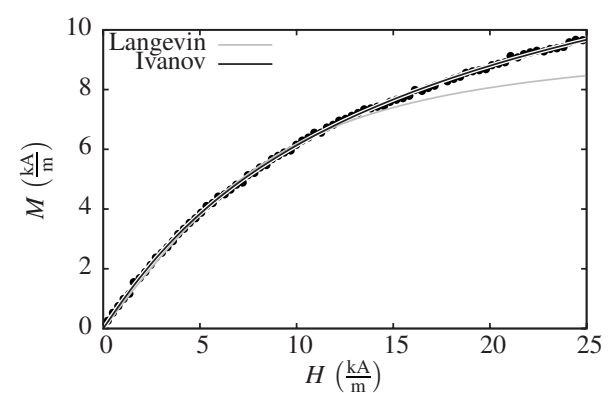

FIG. 5. Nonlinear magnetization curve $M(H)$. Black solid circles: measured data; black line: fit using the Ivanov model [17]; gray line: fitted Langevin function.

$\mathrm{mm}$ of the vessel. The gray symbols give the longitudinal cut for a driving to the right-hand side. The liquid ramp can be approximated by

$$
h(x)=\tan (\gamma) x+h_{0},
$$

as indicated by the fitted lines. The difference of the inclinations is measured by the angle $2 \gamma$. This value is representative for the pressure gradient created by the magnetic pump.

\section{ESTIMATE OF THE PRESSURE GRADIENT}

The buildup of the liquid ramp is caused by the Kelvin force since we apply an inhomogeneous magnetic field to magnetized matter. The system can be described by the ferrohydrodynamic Bernoulli equation [1]

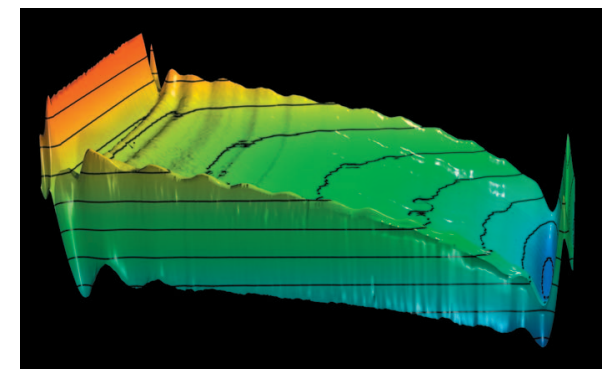

(a)

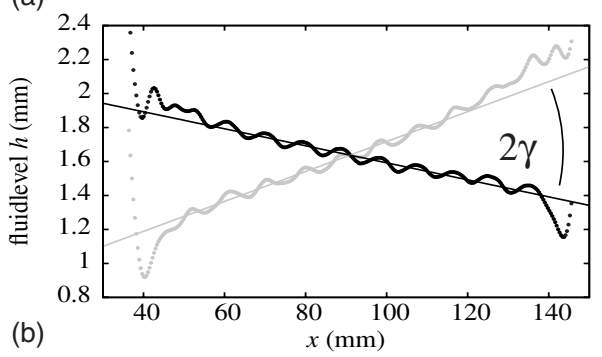

FIG. 6. (Color) A liquid ramp: (a) three-dimensional reconstruction. The height difference between three subsequent black lines is $1.0 \mathrm{~mm}$. (b) Averaged height profiles for forcing to the left-hand side (black) and to the right-hand side (gray). The solid lines represent fits by Eq. (5) within the range between $x=60 \mathrm{~mm}$ and $x$ $=120 \mathrm{~mm}$.

$$
\rho \frac{\partial \vec{v}}{\partial t}+\vec{v} \cdot \vec{\nabla} \vec{v}=-\vec{\nabla} p+\mu_{0} M \vec{\nabla} H+\eta \vec{\nabla}^{2} \vec{v}+\rho \vec{g} .
$$

For the modeling, we assume our system to contain no flow; thus, all the terms with $v$ vanish. Further, we use a onedimensional ansatz and get

$$
\frac{\partial p}{\partial x}=\mu_{0} M_{\mathrm{eff}} \frac{\partial H_{G}}{\partial x} .
$$

Here, the gravity term vanishes, because $\vec{g}$ is perpendicular to $\vec{x}$. The strength $H_{G}$ of the external magnetic field in harmonic approximation is given by Eq. (1). The effective magnetization is defined as

$$
M_{\text {eff }}(x)=M \frac{h(x)}{h_{0}},
$$

where $h_{0}$ denotes the average height of the ferrofluid layer, and $M$ is assumed to be constant. Assuming a harmonic wave for the height modulation

$$
h(x)=h_{0}+\Delta h \sin \left(\omega t-k_{G} x+\phi\right),
$$

where $\phi$ denotes the phase difference with respect to the magnetic field, one gets

$$
\begin{aligned}
M_{\text {eff }}(x)= & M_{\text {eff,ip }} \sin \left(\omega t-k_{G} x\right)-M_{\text {eff,op }} \cos \left(\omega t-k_{G} x\right) \\
& +M_{\text {eff }, 0} .
\end{aligned}
$$

Here, $M_{\text {eff,ip }}$ denotes the part of the magnetization which is in phase with the external field according to Eq. (1). In contrast, $M_{\text {eff,op }}$ is out of phase with $H_{G}$, but in phase with the gradient

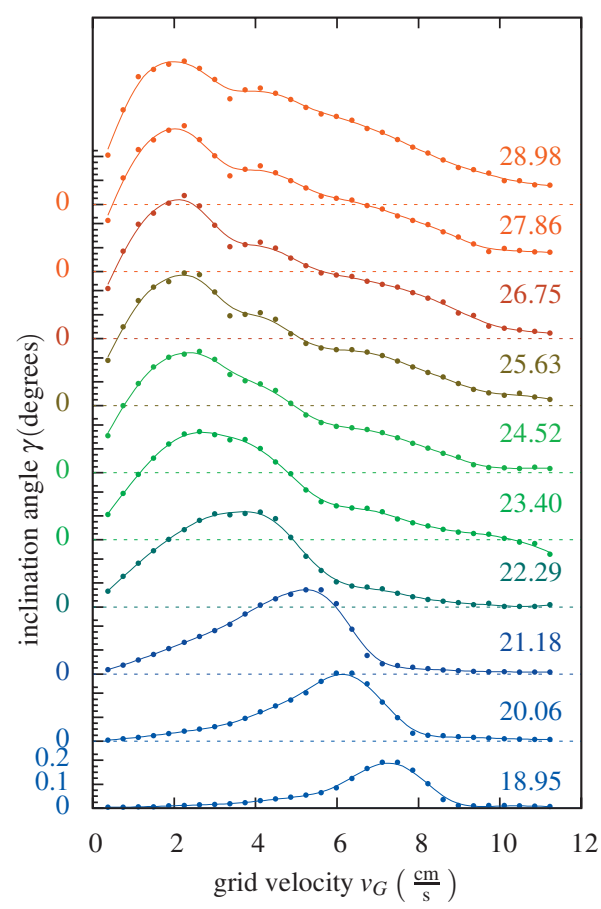

FIG. 7. (Color) Selection of surface inclinations versus the driving frequency for different $\mu_{0} H_{0}$ (denoted by the right ordinate in $\mathrm{mT})$; the curves are shifted equidistantly upward to avoid intersections. The data points are connected by splines to guide the eye. 


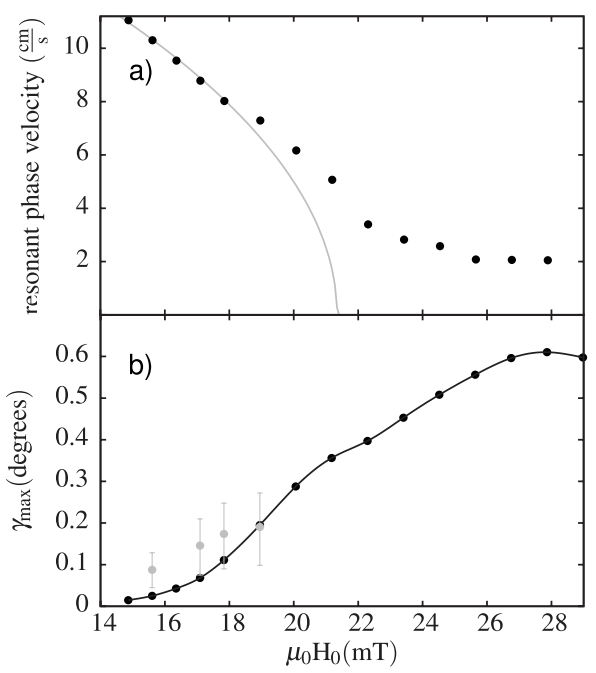

FIG. 8. (a) Resonant phase velocity versus applied magnetic field. The black solid circles give the experimental results. The gray line stems from Eq. (14); (b) angles of maximal surface inclinations over the applied magnetic field. Black symbols are experimental data points. The black line just connects the points via a spline fit to guide the eyes. The gray circles are calculated according to Eq. (15).

$$
\frac{\partial H_{G}(x, t)}{\partial x}=-k \Delta H_{G} \cos \left(\omega t-k_{G} x\right)
$$

of the external field. Inserting $M_{\text {eff }}$ from Eq. (10) into Eq. (7) and averaging over one period of the external field leads to a mean pressure gradient,

$$
\left\langle\frac{\partial p}{\partial x}\right\rangle_{x}=\frac{\int_{0}^{\lambda} \mu_{0} M_{\mathrm{eff}} \frac{\partial H_{G}}{\partial x} d x}{\lambda},
$$

along the $x$ direction. Due to the free surface of the fluid in the experiment, we can read off the corresponding pressure gradient of the one-dimensional model directly. It can be determined by the measured inclination angle $\gamma$ of the surface as $\rho g \tan \gamma$.

\section{EXPERIMENTAL RESULTS}

In order to characterize the pressure difference created by the pump, we investigate its dependence on the externally applied field $H_{0}$ and the belt velocity $v_{G}$. Figure 7 presents the results for ten different values of $H_{0}$. For each field strength, $v_{G}$ was raised from 0 to $11.2 \mathrm{~cm} / \mathrm{s}$ in 30 steps.

Although the width and the shape of the $\gamma(v)$ curves change with the applied field, in each case the pressure difference exhibits a maximum at a certain driving velocity $v_{m}\left(H_{0}\right)$, e.g., for $\mu_{0} H_{0}=18.95 \mathrm{mT} \gamma$ reaches a maximum at $7.29 \mathrm{~cm} / \mathrm{s}$. With increasing $H_{0}$ this maximum is shifted to lower velocities. In order to determine the position of the maxima $\left(v_{m}\right)$, we calculate the barycenter of those data being larger than $75 \%$ of the maximal value of $\gamma$.

In Fig. 8(a) the outcome is marked by black solid circles. The gray curve denotes a model presented in the next sec- tion. The maximal surface inclination is extracted by fitting parabolas to the upper part of the curves. The result is plotted in Fig. 8(b). It demonstrates a monotonic increase in the efficiency of the pump with the applied magnetic field, which seems to saturate at about $30 \mathrm{mT}$.

\section{MODELING THE RESONANCE}

The resonance of $\gamma$ for a specific $v_{m}$, as depicted in Fig. 7, resembles the resonance of the amplitudes of surface waves below the onset of the Rosensweig instability $[13,14]$. The resonant phase velocity $v_{p}\left(H_{0}\right)$ can be captured by the dispersion relation for an inviscid magnetic layer of infinite depth [14].

According to Eq. (12), we expect a monotonic relation between the wave amplitudes and the inclination $\gamma$. The function $v_{m}\left(H_{0}\right)$ in Fig. 8(a) can be modeled in a certain regime by utilizing the dispersion relation for small surface wave amplitudes,

$$
\omega^{2}(k)=g_{\mathrm{e}} k+\frac{\sigma}{\rho} k^{3}-\frac{\mu_{0}}{\rho} \frac{r}{r+1} k^{2} M^{2} .
$$

It can be deduced from Eq. (36) in Ref. [20], as detailed in Ref. [21]. The effective permeability $r$ is the geometric mean $r=\sqrt{\left(1+\chi_{\mathrm{ta}}\right)\left(1+\chi_{\mathrm{ch}}\right)}$, where $\chi_{\mathrm{ta}}=(\partial M / \partial H)$ denotes the tangential susceptibility and $\chi_{\mathrm{ch}}=(M / H)$ denotes the chord susceptibility [1]. For the resonant phase velocity at $k=k_{G}$ one yields

$$
v_{p}=\frac{\omega}{k_{G}}=\sqrt{\frac{g_{\mathrm{e}}}{k_{G}}+\frac{\sigma}{\rho} k_{G}-\frac{\mu_{0}}{\rho} \frac{r}{r+1} M^{2}} .
$$

Using the jump condition for the field at the bottom of the container $H_{\text {ext }}=H_{i}+M\left(H_{i}\right)$ and assuming that $H_{\text {ext }}$ is approximately given by $H_{0}$, one can obtain $v_{p}\left(H_{0}\right)$ numerically. The outcome is marked in Fig. 8(a) by the solid line. It has been calculated using the magnetization curve as presented in Fig. 5. It shows a good agreement with the measured data only for small values of the field, because it is based on the assumption of small amplitudes of the surface waves. The intersection of this line with the $x$ axis at $\mu_{0} H_{\mathrm{c} \text {,calc }}$ $=21.35 \mathrm{mT}$ denotes the estimate for the onset of the Rosensweig instability, where surface deformations spontaneously form without any external modulation. This critical value has been independently measured (see Sec. II and Table I) and matches within the experimental uncertainty.

The maximum of the pressure gradient has experimentally been observed for driving at $v_{m}$ as shown in Fig. 8(b). The phase shift $\phi$ between $H_{G}$ and $M_{\text {eff }}$ is assumed to be $\frac{\pi}{2}$ for this resonant driving, in analogy to a driven harmonic oscillator. Using Eq. (12) and the small-angle approximation $\tan \gamma=\gamma$ this assumption leads to

$$
\gamma=\frac{\mu_{0} M \Delta H_{G} k \Delta h}{2 \rho g h_{0}} .
$$

We measured $\Delta h$ with a laser reflection method, as described in Ref. [14]. When using this method, we assume a sinusoidal modulation of the surface; it is thus restricted to 
small values of the magnetic field $H_{0}$. Therefore, we are restricted to values well below $H_{c \text {,nonlin }}$. The corresponding data points for four measured values of $\Delta h$ are indicated in Fig. 8(b) by circles. The data only agree within about $50 \%$. This is partly due to (i) the flutter in $\lambda_{G}$, (ii) the geometrical uncertainty connected with the laser reflection method, (iii) the variation in $h_{0}$ along the ramp, (iv) the variation of the magnetization and the field modulation within the layer thickness, and $(v)$ the neglect of the higher harmonics of $h(x, t)$. All these uncertainties are taken into account by the error bars. Moreover, the model does not take into account any backflow effects and assumes a strictly two-dimensional geometry.

\section{CONCLUSION}

In this paper we have demonstrated a pump for magnetic fluids which is based on a resonance phenomenon in the advent of the Rosensweig instability. Our driving relies fundamentally on the excitation of surface waves. It is thus restricted to open channel geometries and differs substantially from "ferrohydrodynamic pumping" as described in Ref. [8] which works in closed channels. That method, however, is most effective for frequencies comparable to the inverse Brownian relaxation time $(\approx 10 \mathrm{kHz})$, while our driving mechanism favors low frequencies $(\approx 10 \mathrm{~Hz})$.

We have characterized the pump under no-load conditions by measuring the static pressure gradient via a radioscopic method. Under variation of the driving velocity, the pressure gradient shows a resonance. The resonant phase velocity decreases with the applied magnetic field. This can be understood by calculating the phase velocity of surface waves from the dispersion relation, taking into account the nonlinear magnetization curve. The pressure gradient is observed to increase with the field, which is captured by a two- dimensional model derived from the ferrohydrodynamic Bernoulli equation assuming a static equilibrium. This ansatz leads to a transport effect, whose strength grows linearly with the surface modulation; the peristaltic pumping on the other hand grows with its square. A refined model should include both effects and would be a task for numerical investigations.

In future work, the spatiotemporal driving of the liquid could be achieved in different ways. While in the present study we used a moving belt, harboring iron rods, to drive the liquid, alternatively the driving may be realized by a network of current carrying wires without any moving parts. This would allow us to control the modulation amplitude and the bias of the magnetic field independently.

The pump has been tested under no-load conditions. The opposite approach would be to characterize the pump under maximum-load conditions. Therefore, the input and output of the pump must be connected by an open duct with minimal flow resistance, similar to the setup described in Ref. [9]. Note that our resonant driving may be exploited as well to drive nonmagnetic liquids via the magnetically driven peristaltic motion investigated in Ref. [22].

So far we have focused our measurements on the resonant behavior below the formation of surface undulations. It remains to be investigated how the spatiotemporal driving interacts with the secondary instabilities leading to more complicated surface patterns [23].

\section{ACKNOWLEDGMENTS}

The authors are grateful to A. Beetz and C. Groh for their contributions to the experimental design and K. Oetter for the mechanical realization. Further we want to thank K. Zimmermann and A. Naletova for discussion and DFG SFB 481 for financial support.
[1] R. E. Rosensweig, Ferrohydrodynamics (Cambridge University Press, Cambridge, England, 1985).

[2] M. I. Shliomis and K. I. Morozov, Phys. Fluids 6, 2855 (1994).

[3] J.-C. Bacri, R. Perzynski, M. I. Shliomis, and G. I. Burde, Phys. Rev. Lett. 75, 2128 (1995).

[4] A. Zeuner, R. Richter, and I. Rehberg, Phys. Rev. E 58, 6287 (1998).

[5] R. Moskowitz and R. Rosensweig, Appl. Phys. Lett. 11, 301 (1967).

[6] R. Krauß, M. Liu, B. Reimann, R. Richter, and I. Rehberg, Appl. Phys. Lett. 86, 024102 (2005).

[7] L. D. Mao and H. Koser, J. Magn. Magn. Mater. 289, 199 (2005).

[8] L. D. Mao and H. Koser, Nanotechnology 17, S34 (2006).

[9] H. Kikura, T. Sawada, T. Tanahashi, and L. Seo, J. Magn. Magn. Mater. 85, 167 (1990).

[10] K. Zimmermann, I. Zeidis, V. A. Naletova, and V. A. Turkov, J. Magn. Magn. Mater. 268, 227 (2004).

[11] M. D. Cowley and R. E. Rosensweig, J. Fluid Mech. 30, 671 (1967).
[12] B. Reimann, R. Richter, I. Rehberg, and A. Lange, Phys. Rev. E 68, 036220 (2003).

[13] V. G. Bashtovoi and M. S. Krakov, Magnetohydrodynamics 13, 17 (1977).

[14] A. Beetz, C. Gollwitzer, R. Richter, and I. Rehberg, J. Phys.: Condens. Matter 20, 204109 (2008).

[15] R. Richter and J. Bläsing, Rev. Sci. Instrum. 72, 1729 (2001).

[16] C. Gollwitzer, G. Matthies, R. Richter, I. Rehberg, and L. Tobiska, J. Fluid Mech. 571, 455 (2007).

[17] A. O. Ivanov and O. B. Kuznetsova, Phys. Rev. E 64, 041405 (2001).

[18] R. Richter and I. V. Barashenkov, Phys. Rev. Lett. 94, 184503 (2005).

[19] R. Friedrichs and A. Engel, Phys. Rev. E 64, 021406 (2001).

[20] R. E. Zelazo and J. R. Melcher, J. Fluid Mech. 39, 1 (1969).

[21] B. Reimann, R. Richter, H. Knieling, R. Friedrichs, and I. Rehberg, Phys. Rev. E 71, 055202(R) (2005).

[22] G. Park and S. Park, IEEE Trans. Magn. 35, 4058 (1999).

[23] S. Rüdiger, E. M. Nicola, J. Casademunt, and L. Kramer, Phys. Rep. 447, 73 (2007). 